\title{
Article
}

\section{TF-RBP-AS Triplet Analysis Reveals the Mechanisms of Aberrant Alternative Splicing Events in Kidney Cancer: Implications for Their Possible Clinical Use as Prognostic and Therapeutic Biomarkers}

\author{
Meng He and Fuyan $\mathrm{Hu} *$ (D) \\ Department of Statistics, School of Science, Wuhan University of Technology, 122 Luoshi Road, \\ Wuhan 430070, China; hm798104845@whut.edu.cn \\ * Correspondence: fuyanhu@whut.edu.cn; Tel.: +86-027-87108033
}

check for updates

Citation: He, M.; Hu, F. TF-RBP-AS Triplet Analysis Reveals the Mechanisms of Aberrant Alternative Splicing Events in Kidney Cancer: Implications for Their Possible Clinical Use as Prognostic and Therapeutic Biomarkers. Int. J. Mol. Sci. 2021, 22, 8789. https:// doi.org/10.3390/ijms22168789

Academic Editor: Ronald Moura

Received: 10 June 2021

Accepted: 11 August 2021

Published: 16 August 2021

Publisher's Note: MDPI stays neutral with regard to jurisdictional claims in published maps and institutional affiliations.

Copyright: (C) 2021 by the authors Licensee MDPI, Basel, Switzerland. This article is an open access article distributed under the terms and conditions of the Creative Commons Attribution (CC BY) license (https:// creativecommons.org/licenses/by/ $4.0 /)$.

\begin{abstract}
Aberrant alternative splicing (AS) is increasingly linked to cancer; however, how AS contributes to cancer development still remains largely unknown. AS events (ASEs) are largely regulated by RNA-binding proteins (RBPs) whose ability can be modulated by a variety of genetic and epigenetic mechanisms. In this study, we used a computational framework to investigate the roles of transcription factors (TFs) on regulating RBP-AS interactions. A total of 6519 TF-RBPAS triplets were identified, including 290 TFs, 175 RBPs, and 16 ASEs from TCGA-KIRC RNA sequencing data. TF function categories were defined according to correlation changes between RBP expression and their targeted ASEs. The results suggested that most TFs affected multiple targets, and six different classes of TF-mediated transcriptional dysregulations were identified. Then, regulatory networks were constructed for TF-RBP-AS triplets. Further pathway-enrichment analysis showed that these TFs and RBPs involved in triplets were enriched in a variety of pathways that were associated with cancer development and progression. Survival analysis showed that some triplets were highly associated with survival rates. These findings demonstrated that the integration of TFs into alternative splicing regulatory networks can help us in understanding the roles of alternative splicing in cancer.
\end{abstract}

Keywords: alternative splicing; RNA-binding protein; transcription factor; TF-RBP-AS triplets; regulation mechanism

\section{Introduction}

Renal cell carcinoma (RCC) is a common malignant tumor that, according to 2020 global cancer data released by the International Agency for Research on Cancer (IARC), accounts for $2.2 \%$ of all new cancer cases, with approximately 431,288 new cases and 179,368 deaths worldwide, and there will be approximately 73,587 new cases and 43,196 deaths in China. There are different types of RCC, and kidney renal clear cell carcinoma (KIRC) is the most common type of RCC, accounting for about 75\% of adult RCC malignancies [1]. Since $\mathrm{KIRC}$ is radiotherapy- and chemotherapy-resistant, surgery is currently the most effective treatment [2]. Despite early surgical treatment, $30 \%$ of patients with a localized tumor eventually develop metastases, and the five-year overall survival rate of metastatic KIRC is only $12 \%$ [2-4]. Although immune-checkpoint and targeted therapeutics inhibitors have changed the landscape of treatment for KIRC, most patients have never experienced significant clinical benefits $[5,6]$. Therefore, it is essential to reveal the underlying molecular mechanisms of KIRC and find more powerful diagnostic biomarkers or therapeutic targets.

Alternative splicing (AS) is a pivotal process that increases the diversity of proteins [7]. The destruction of this process may lead to disorders of normal cell functions and eventually develop cancer. The dysregulation of alternative splicing is a new hallmark of cancer 
and can be used as a biomarker for drug therapy $[8,9]$. The regulation of alternative splicing is a complicated process, including cis-regulatory elements and trans-acting factors [10,11]. Growing evidence indicates that alternative splicing is strongly associated with kidney cancer. For example, some specific alternative splicing events (ASEs) are potential prognostic biomarkers of kidney cancer [12], some ASEs were predicted to be associated with cancer stemness in KIRC [13], and a novel prognostic index based on prognosis-related AS events was revealed in KIRC [14]. However, comprehensively understanding the alterations of AS in KIRC remains unknown. Thus, exploring the regulatory patterns that control AS provides valuable molecular insights and provides solutions for cancer treatment [15].

ASEs are largely regulated by RNA-binding proteins (RBPs) that can bind to cisregulatory elements in introns and exons to regulate splicing [16]. Specifically, RBPs recruit different factors and enzymes to form different complexes that bind to specific regulatory sequences of their target pre-mRNA, thus modulating RNA alternative splicing $[17,18]$. However, the mechanism of interactions between splicing complexes and target pre-mRNA is complex. An RBP can act on hundreds of mRNA target genes, but under the influence of environmental stimuli, an RBP only acts on a subset of its RNA target genes [15], which is often influenced by transcription factors (TFs). By regulating the activity of RBP, TFs can affect downstream alternative splicing outcomes. Therefore, discovering and elucidating the interactions between RBPs, AS events (ASEs), and TFs are necessary for understanding the mechanisms of alternative splicing.

AS could be mediated by nonsense-mediated mRNA decay (NMD), which is an mRNA surveillance pathway in eukaryotic cells that can rapidly degrade mRNAs bearing premature translation termination codon (PTC) to protect cells from adverse effects of truncated proteins $[19,20]$. Some studies showed that AS coupled to NMD (AS-NMD) as a novel post-transcriptional mechanism is closely related to cancer [21,22]. Thus, studying AS-NMD may provide a novel view on understanding AS mechanisms in kidney cancer.

In this study, we established a computational method for studying the relationships between RBPs, ASEs, and TFs. Our method discovers how alternative splicing outcomes change under the regulation of the same RBP when transcription factor expression is different. A triplet contains three elements: a specific ASE, a specific RBP that may regulate the ASE, and a TF that may change the splicing regulation of the RBP. We first used the TCGA-KIRC dataset to analyze and select key cancer-specific alternative splicing, and differentially expressed RBPs and TFs in cancer; then, we applied linear mixed models to identify triplets in kidney cancer. A regulatory network for triplets in KIRC was established, and the potential mechanisms were explored. Survival analysis showed that these triplets were highly associated with survival rates. The results provided another perspective for further investigations into the molecular pathogenesis of kidney cancer and the selection of the potential therapeutic targets for the treatment of kidney cancer.

\section{Results}

\subsection{Screening of Key ASEs in Kidney Cancer}

The file of ASEs was composed of PSI values of 46,415 ASEs involved 10,600 genes for 533 KIRC patients and 77 normal samples. According to their splicing pattern, these ASEs were divided into seven types: exon skip (ES), mutually exclusive exons (ME), retained intron (RI), alternate promoter (AP), alternate terminator (AT), alternate donor site (AD), and alternate acceptor site (AA), which are illustrated in Figure 1A. Furthermore, an UpSet plot was generated to visualize the intersecting sets of each AS type, as shown in Figure 1B. 
A

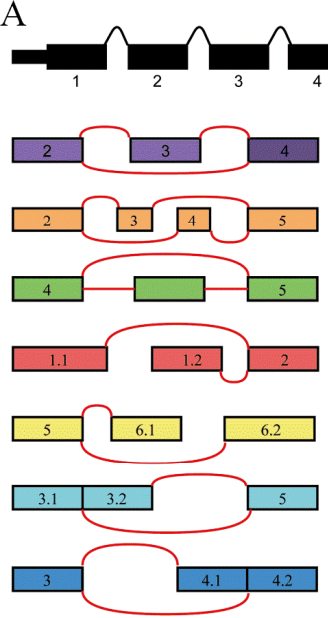

$\mathrm{D}$

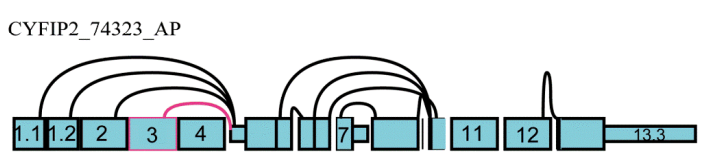

PCNA_58648_AP
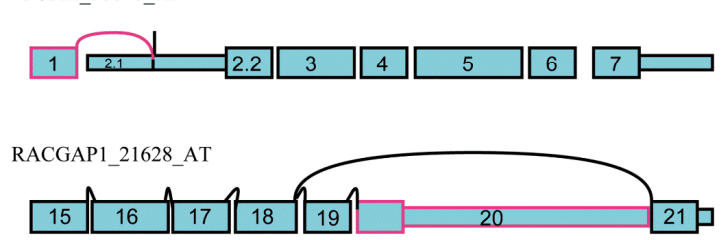

TMEM213_81931_AT

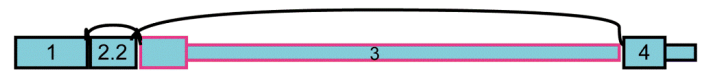

SLC17A3_75564_ES
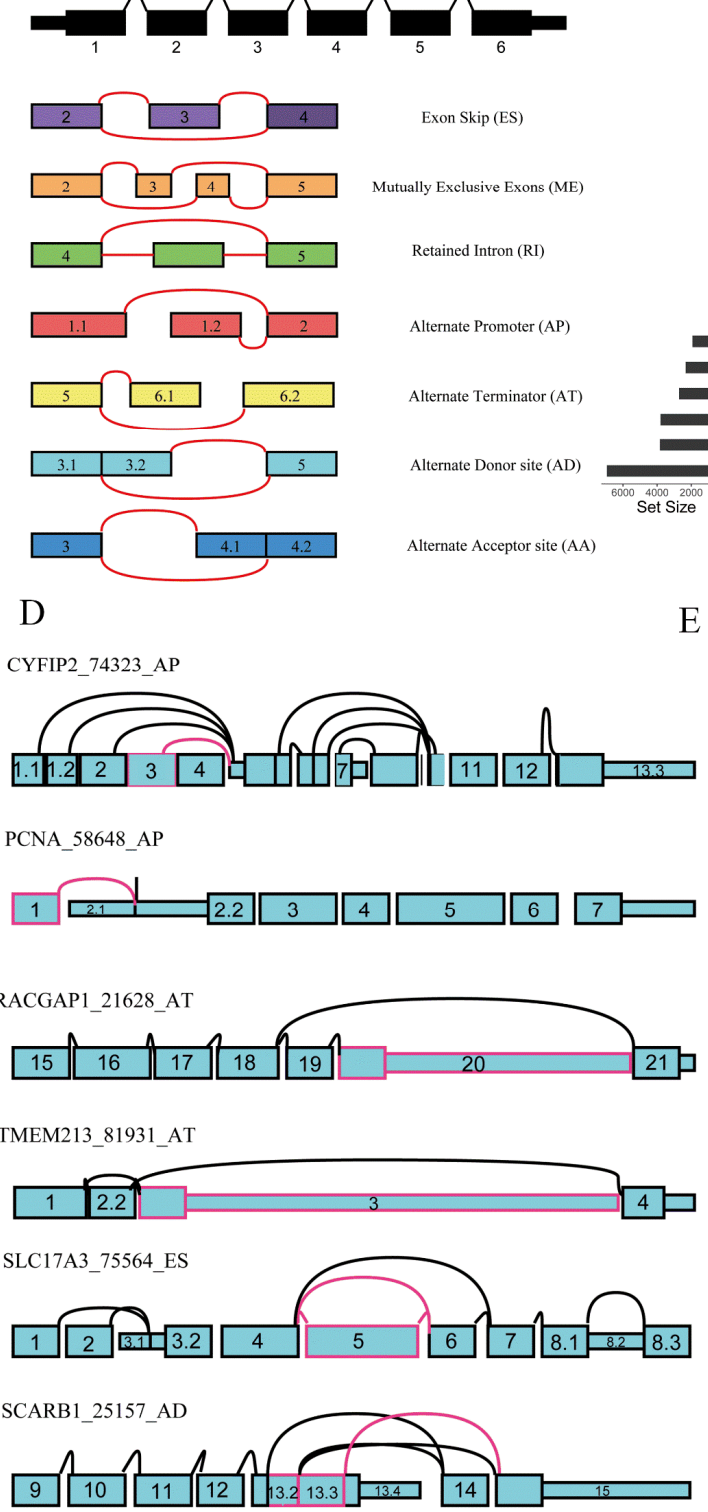

$\mathrm{E}$
B
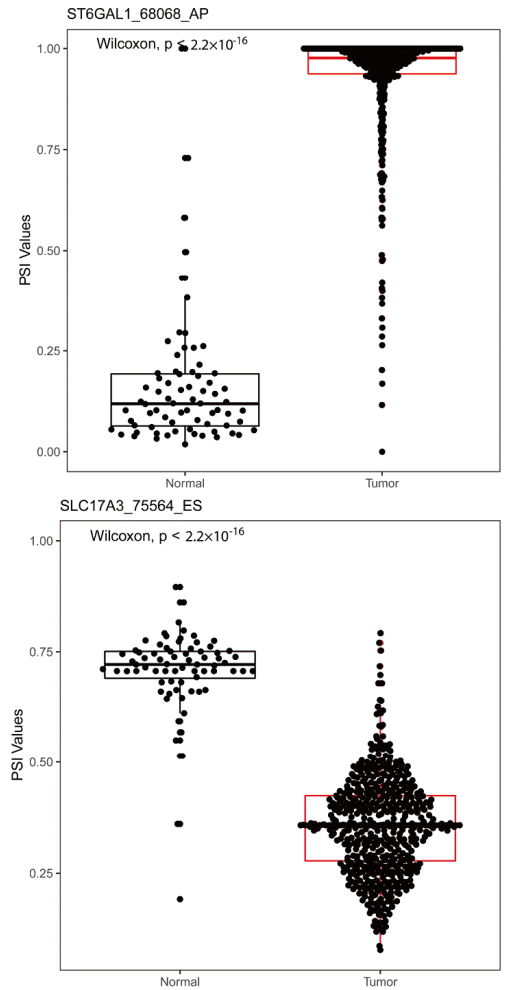

$\mathrm{C}$
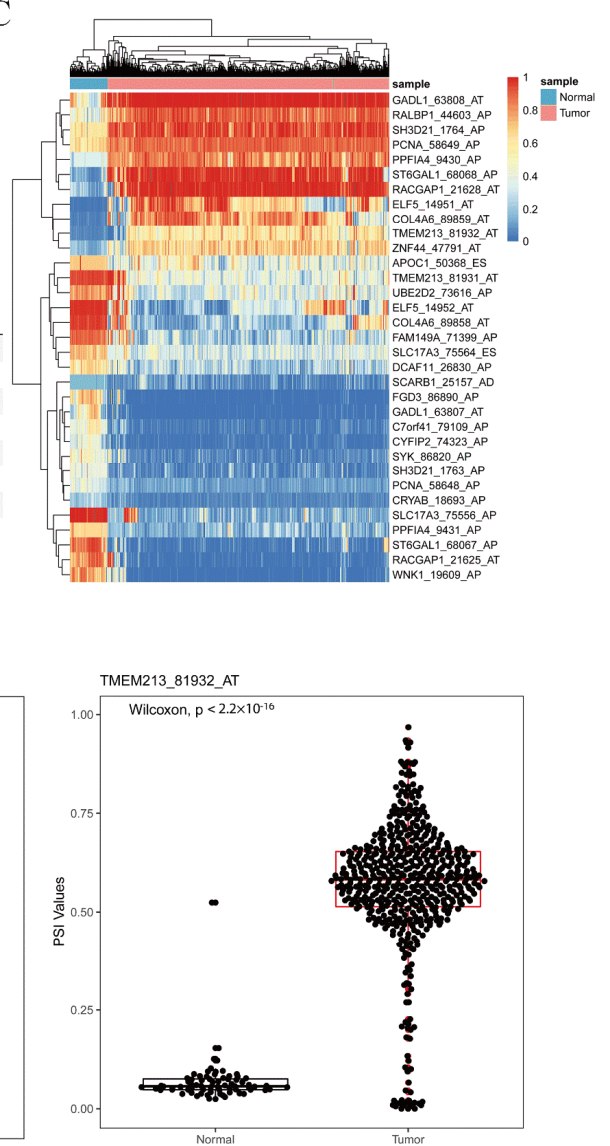

SCARB1_25157_AD

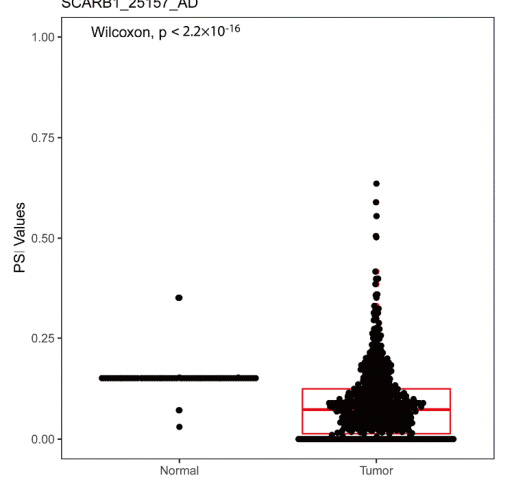

Figure 1. Overview of AS events profiling in KIRC. (A) Seven types of AS events: exon skip (ES), mutually exclusive exons $(\mathrm{ME})$, retained intron $(\mathrm{RI})$, alternate promoter $(\mathrm{AP})$, alternate terminator $(\mathrm{AT})$, alternate donor site (AD), and alternate acceptor site (AA). (B) UpSet plot of interactions between the seven types of detected AS events $(\mathrm{n}=46,415)$ in KIRC. (C) Heat map of significant ASEs $(n=33)$. Horizontal axis shows clustering information of samples (normal or tumor); left longitudinal axis shows clustering information of ASEs. Gradual change in color from green to red represents PSI value of ASEs altered from low to high. (D) Splice graphs of some representative ASEs. Exons were drawn to scale, and connecting arcs represent splice paths. (E) Boxplots of four differentially expressed ASEs showing different expressions of AS events between KIRC and normal samples. Wilcoxon test was used for data comparison.

By comparing the expression of ASE in KIRC and normal samples, 33 ASEs were identified as differentially expressed ASE through the iterative MI-SIS method, among which there were 19 APs, 2 ESs, 11 ATs, and 1 AD. Detailed information on the differentially expressed ASEs is listed in Table 1. A receiver-operating-characteristic (ROC) curve was drawn to show the prediction accuracy of our method, as shown in Supplementary Figure S1, which suggested that our method reached a high area under the curve 
(AUC $=0.993$. . A heat map was used to elaborate the expression differences of the 33 differentially expressed ASEs between the tumor and normal samples, as shown in Figure 1C. Some of the differentially expressed ASEs are depicted as splice graphs, which summarize the transcript variations into directed acyclic graphs, and represent exons as rectangular nodes and splice junctions as edges (Figure 1D). Furthermore, the boxplots of four differentially expressed ASEs are shown in Figure 1E. Both the heat map and boxplots suggested that the 33 differentially expressed ASEs could be used as diagnostic biomarkers for KIRC.

Table 1. Detailed information of 33 cancer-specific ASEs.

\begin{tabular}{|c|c|c|c|c|c|c|c|c|}
\hline Symbol & Gene & AS Type & Exons & From Exon & To Exon & Mean_T & Mean_N & Mi Value \\
\hline \multicolumn{9}{|l|}{ Upregulated } \\
\hline PPFIA4_9430_AP & PPFIA4 & $\mathrm{AP}$ & 19.1 & & & 0.816 & 0.345 & 0.354 \\
\hline ST6GAL1_68068_AP & ST6GAL1 & $\mathrm{AP}$ & 1 & & & 0.932 & 0.161 & 0.347 \\
\hline RACGAP1_21628_AT & RACGAP1 & AT & 20 & & & 0.944 & 0.216 & 0.341 \\
\hline TMEM213_81932_AT & TMEM213 & AT & 4 & & & 0.565 & 0.070 & 0.340 \\
\hline SH3D21_1764_AP & SH3D21 & $\mathrm{AP}$ & 4.1 & & & 0.924 & 0.600 & 0.338 \\
\hline GADL1_63808_AT & GADL1 & $\mathrm{AT}$ & 12.2 & & & 0.986 & 0.549 & 0.338 \\
\hline ELF5_14951_AT & ELF5 & AT & 4.2 & & & 0.648 & 0.026 & 0.336 \\
\hline PCNA_58649_AP & PC & $\mathrm{AP}$ & 2.1 & & & 0.884 & 0.631 & 0.333 \\
\hline ZNF44_47791_AT & ZNF44 & $\mathrm{AT}$ & 6.2 & & & 0.652 & 0.177 & 0.328 \\
\hline RALBP1_44603_AP & RALBP1 & $\mathrm{AP}$ & 2 & & & 0.878 & 0.455 & 0.325 \\
\hline $\begin{array}{c}\text { COL4A6_89859_AT } \\
\text { Downregulated }\end{array}$ & COL4A6 & AT & 52 & & & 0.708 & 0.054 & 0.322 \\
\hline PPFIA4_9431_AP & PPFIA4 & $\mathrm{AP}$ & 1 & & & 0.180 & 0.655 & 0.354 \\
\hline ST6GAL1_68067_AP & ST6GAL1 & $\mathrm{AP}$ & 3 & & & 0.068 & 0.839 & 0.347 \\
\hline C7orf41_79109_AP & C7orf41 & $\mathrm{AP}$ & 2 & & & 0.047 & 0.470 & 0.346 \\
\hline FGD3_86890_AP & FGD3 & $\mathrm{AP}$ & 3 & & & 0.019 & 0.589 & 0.342 \\
\hline RACGAP1_21625_AT & RACGAP1 & AT & 21 & & & 0.056 & 0.784 & 0.341 \\
\hline TMEM213_81931_AT & TMEM213 & $\mathrm{AT}$ & 3 & & & 0.435 & 0.931 & 0.340 \\
\hline WNK1_19609_AP & WNK1 & $\mathrm{AP}$ & 5 & & & 0.036 & 0.736 & 0.340 \\
\hline SH3D21_1763_AP & SH3D21 & $\mathrm{AP}$ & 1 & & & 0.076 & 0.408 & 0.339 \\
\hline GADL1_63807_AT & GADL1 & $\mathrm{AT}$ & 15 & & & 0.014 & 0.451 & 0.338 \\
\hline ELF5_14952_AT & ELF5 & AT & 8 & & & 0.352 & 0.974 & 0.336 \\
\hline PCNA_58648_AP & PC & $\mathrm{AP}$ & 1 & & & 0.116 & 0.369 & 0.333 \\
\hline SLC17A3_75564_ES & SLC17A3 & ES & 5 & 4 & 6 & 0.358 & 0.707 & 0.331 \\
\hline APOC1_50368_ES & APOC1 & ES & $4: 5.1$ & 3.2 & 7 & 0.424 & 0.692 & 0.331 \\
\hline SCARB1_25157_AD & SCARB1 & $\mathrm{AD}$ & 13.3 & 13.2 & 15 & 0.089 & 0.151 & 0.326 \\
\hline SYK_86820_AP & SYK & $\mathrm{AP}$ & 2 & & & 0.079 & 0.456 & 0.324 \\
\hline SLC17A3_75556_AP & SLC17A3 & $\mathrm{AP}$ & 2 & & & 0.199 & 0.970 & 0.323 \\
\hline UBE2D2_73616_AP & UBE2D2 & $\mathrm{AP}$ & 2 & & & 0.395 & 0.780 & 0.322 \\
\hline COL4A6_89858_AT & COL4A6 & $\mathrm{AT}$ & 51.2 & & & 0.292 & 0.946 & 0.322 \\
\hline DCAF11_26830_AP & DCAF11 & $\mathrm{AP}$ & 2.1 & & & 0.262 & 0.621 & 0.321 \\
\hline CYFIP2_74323_AP & CYFIP2 & $\mathrm{AP}$ & 3 & & & 0.030 & 0.371 & 0.319 \\
\hline CRYAB_18693_AP & CRYAB & $\mathrm{AP}$ & 5.1 & & & 0.060 & 0.224 & 0.318 \\
\hline FAM149A_71399_AP & FAM149A & $\mathrm{AP}$ & 3 & & & 0.288 & 0.867 & 0.318 \\
\hline
\end{tabular}

\subsection{Identification of Differentially Expressed RBPs and TFs in Kidney Cancer}

The method of $\log 2 \mathrm{FC}$ and the Wilcoxon test were used to identify differentially expressed TFs and RBPs from 1635 RBPs and 1826 TFs. Lastly, 302 TFs and 177 RBPs were selected under the criteria of $p$ value $<0.05$ and $|\log 2 \mathrm{FC}|>1$. All are shown in Supplementary Tables S1 and S2.

\subsection{Category of TF Action}

Depending on whether the correlation of RBP-AS increased or decreased in two "abundance" groups (low to high) of TFs, three possible modes of TFs action were identified: "attenuates interaction", "enhances interaction", and "inverts interaction". Among them, each mode comprised two subtypes: strengthen attenuation interaction (SAI), weaken 
attenuation interaction (WAI); strengthen enhancement interaction (SEI), weaken enhancement interaction (WEI); and invert positive to negative (IPN), invert negative to positive (INP). These cases and details interpretations are listed in Table 2.

Table 2. Categories of TF-mediated RBP regulations on target AS.

\begin{tabular}{|c|c|c|c|c|}
\hline Pattern & PCC $_{\text {low }}$ & PCC $_{\text {high }}$ & $\triangle \mathrm{PCC}$ & Subtype Mode \\
\hline Enhances & - & - & $\left|\mathrm{PCC}_{\text {low }}\right|<\left|\mathrm{PCC}_{\text {high }}\right|$ & Strengthens attenuation interaction (SAI) \\
\hline Attenuates & 一 & - & $\left|\mathrm{PCC}_{\text {low }}\right|>\left|\mathrm{PCC}_{\text {high }}\right|$ & Weakens attenuation interaction (WAI) \\
\hline Inverts & + & - & & Inverts positive to negative (IPN) \\
\hline Inverts & - & + & & Inverts negative to positive (INP) \\
\hline Enhances & + & ++ & $\left|\mathrm{PCC}_{\text {low }}\right|<\left|\mathrm{PCC}_{\text {high }}\right|$ & Strengthens enhancement interaction (SEI) \\
\hline Attenuates & ++ & + & $\left|\mathrm{PCC}_{\text {low }}\right|>\left|\mathrm{PCC}_{\text {high }}\right|$ & Weakens enhancement interaction (WEI) \\
\hline
\end{tabular}

"+" and " - " signs in the columns indicate positive and negative values of Pearson correlation coefficient, and "++" and "—-" indicate a larger absolute value of Pearson correlation coefficient compared to its control group.

\subsection{Detection of TF-RBP-AS Triplets in Kidney Cancer}

Linear mixed-effects models were employed to identify TF-RBP-AS triplets for differentially expressed TFs $(n=302)$, RBPs $(n=177)$, and ASEs $(n=33)$ based on Formula (1); 6519 TF-mediated significant triplets were statistically significant under our criteria as described in Section 4 (Supplementary Table S3). The 6519 TF-RBP-AS triplets included 290 TFs, 175 RBPs, and 16 ASEs corresponding to 13 genes. According to the correlation changes between RBP and ASE expressions under different abundance groups (low or high) of TF, six subcategories were identified: 688 triplets in SEI, 1018 triplets in WEI, 777 triplets in SAI, 1287 triplets in WAI, 1307 triplets in IPN, and 1442 triplets in INP (Figure 2A).

\subsection{Construction of Splicing-Regulatory Network for Triplets}

We constructed a splicing-regulatory network for 6519 triplets. The splicing-regulatory network was visualized in Cytoscape. The relationships between TF and RBP (purple lines), TF and ASE (green lines), and RBP and ASE (blue lines) are exhibited (Figure 3A). The complexity of alternative splicing regulation can be seen from the triplet network. In addition, we compared this splicing-regulatory network with the known human PPI network in STRING and found that 18 triplets were connected in it as shown in Figure 3B.

In the network of 18 triplets, IRF1, NFKB2, and UBE2D2_73616_AP were the TF, RBP, and ASE with the highest node degree, respectively. Interferon regulatory factor 1 (IRF1) is a tumor-suppressor gene that is associated with RCC, which can promote the apoptosis of tumor cells and increase tumor-cell sensitivity to chemotherapeutic drugs [23]. Nuclear factor kappa B subunit 2 (NFKB2) regulates all important aspects of RCC biology, including resistance to apoptosis, angiogenesis, and multidrug resistance [24]. Ubiquitin conjugating enzyme E2 D2 (UBE2D2) plays an important role in the development of breast cancer [25]. These results showed that the triplet network may play key roles in the development of RCC.

\subsection{Survival and Functional-Enrichment Analyses for Triplets}

To investigate the association between triplets and kidney cancer, we performed survival analysis in tumor samples by constructing prognostic-risk-score models through Formula (2). For each triplet, on the basis of the expression of its components (i.e., an RBP, a TF, and an ASE), each tumor sample had a calculated risk score. Then, all tumor samples were divided into high- and low-risk subgroups with the median value of risk scores as cutoff. Then, survival analysis was carried out for the 6519 triplets, and the results suggested that there were significant survival differences between the high- and low-risk subgroups for 5580 triplets (Supplementary Table S4). For example, although GADL1_63808_AT and UBE2D2_73616_AP were two of the ASEs that did not show too much differential expression, survival analysis indicated that triplets including GADL1_63808_AT and UBE2D2_73616_AP in KIRC were significantly associated with 
overall survival outcomes by comparing the high- and low-risk subgroups (Figure 4A). Survival plots of three representative triplets, namely RUNX2-RPL36-GADL1_63808_AT, MYBL1-RL36-GADL1_63808_AT, and RUNX1-PRSS53-UBE2D2_73616_AP, are shown in Figure $4 \mathrm{~A}$, suggesting that the high-risk subgroup had a worse survival rate for each triplet ( $\log$-rank $p$ values were $9.4 \times 10^{-6}, 0.00019$ and respectively $\left.1.7 \times 10^{-15}\right)$. The results of survival analysis suggested that the detective triplets could serve as prognostic biomarkers for kidney cancer.

A

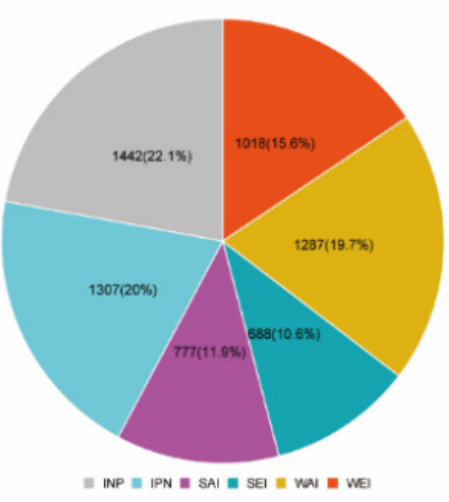

$\mathrm{D}$

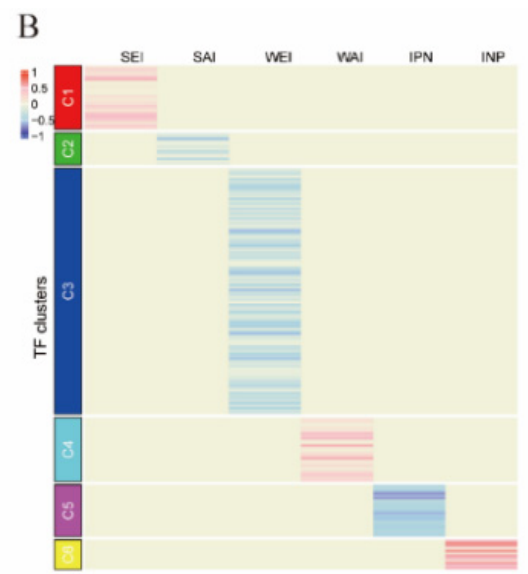

$\mathrm{C}$

E

\begin{tabular}{|c|c|c|c|}
\hline Triplets patterns & ASEs & $\begin{array}{l}\text { RNA binding } \\
\text { procichingars) }\end{array}$ & Tressctiption faction(TFy) \\
\hline SEI & $\begin{array}{l}\text { \& CYFIP2_74323_AP, } \\
\text { UBE2D2 } 73616 \text { AP }\end{array}$ & MAP3K9 & $\begin{array}{l}\text { \% GRHLL, HELT, } \\
\text { ETV4 }\end{array}$ \\
\hline SAI & $\begin{array}{l}\text { eq COLAA6_89859_AT } \\
\text { GADLI_66808_AT }\end{array}$ & MAP3K9 & $\begin{array}{l}\text { e. GRHLZ GSC. } \\
\text { HELT }\end{array}$ \\
\hline wEl & $\begin{array}{l}\text { क PCNM_58649_AP, } \\
\text { RALBPI_46003_AP }\end{array}$ & MAPIK9 & $\begin{array}{l}\text { Ee MAFA SHOX2 } \\
\text { RINX1 }\end{array}$ \\
\hline wAI & $\begin{array}{l}\text { Ee.TMEM213_81932_AT } \\
\text { GADL.1_63808_AT }\end{array}$ & MAPIK9 & $\begin{array}{l}\text { E. ZGLPI, FOXHI, } \\
\text { ARNT2 }\end{array}$ \\
\hline IPN & $\begin{array}{l}\text { eq_RACGAPI_21625_AT } \\
\text { CYFIP_74123_AP }\end{array}$ & MAP3K 9 & $\begin{array}{l}\text { eq BCL.1B, DLXS. } \\
\text { FoxaI }\end{array}$ \\
\hline NPP & $\begin{array}{l}\text { Eg. TMEME13_81932_AT } \\
\text { CYFIP2_,7332_AP }\end{array}$ & MAP3K 9 & $\begin{array}{l}\text { \& DL.X5, FoxCl, } \\
\text { FoxD4 }\end{array}$ \\
\hline
\end{tabular}

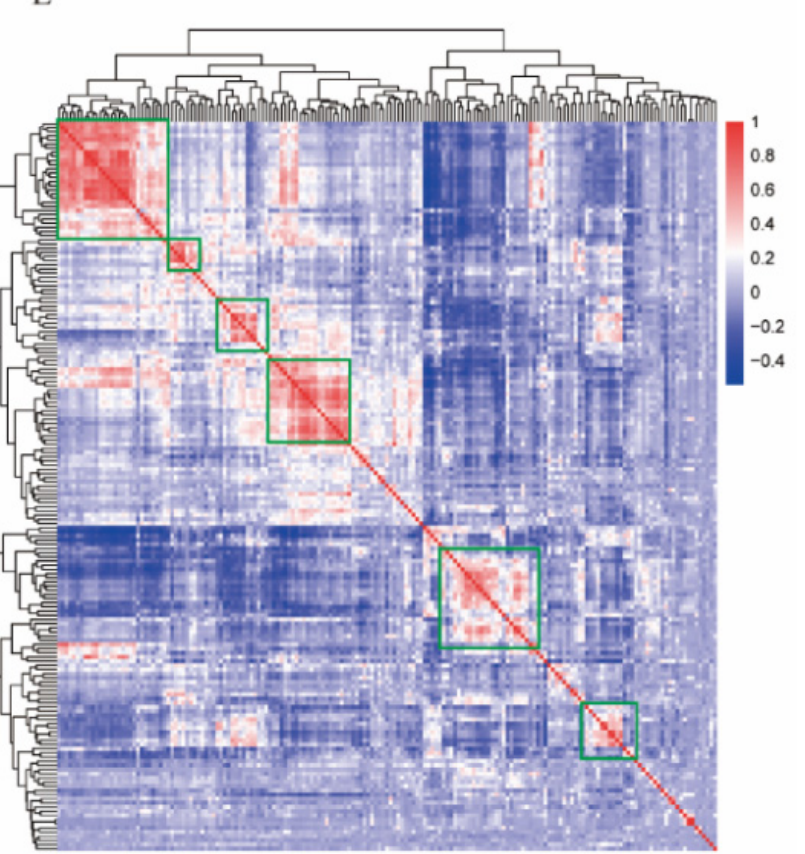

Figure 2. Identified triplets in KIRC. (A) Six mode subcategories of TFs according to correlation between expression of RBP and PSI value of ASE. Number in the pie chart means the percentage of each subcategory. (B) Clusters by the regulation patterns of TFs that are involved in the triplets containing the RBP MAP3K9. Six clusters were grouped according to TF subcategories. (C) Information of some representative triplets involved MAP3K9. (D) Correlation heat map of TFs involved in 6519 triplets. (E) Correlation heat map of RBPs involved in 6519 triplets.

Pathway-enrichment analysis showed that these TFs and RBPs were highly enriched in categories that are associated with cancer development and progression, including herpes simplex virus 1 infection, meiotic cell cycle, and the negative regulation of cell differentiation (Figure 4B,C). In addition, cancer-relevant modulators were identified through a tumor-associated gene list from the Network of Cancer Genes (NCG, v6.0) [26] and the Tumor Suppressor Gene (TSGene, v2.0) databases [27], separately (Figure 4D). The 2378 tu- 
mor diver genes obtained from NCG were 711 known cancer genes and 1667 candidate cancer genes. Among all genes involved in the 6519 triplets, 122 genes overlapped with tumor diver genes, almost reaching $25.58 \%(122 / 477)$ of the total numbers of triplet genes. Approximately $12.37 \%$ (59/477) of the genes were tumor-suppressor genes. These results suggested that these triplets were involved in the occurrence and development of cancer.

\subsection{Analysis of Splicing Event of PCNA_58648_AP Influenced by Triplets in Kidney Cancer}

In this study, 80 triplets were found to be involved in the ASE of PCNA_58648_AP, including 28 TFs and 58 RBPs. Previously studies reported that PCNA had obvious differential expression in RCC and played an important role in cell proliferation [28]. Some triplets of PCNA_58648_AP are shown in Figure 5A.

For example, ETV7 is a modulator of IGF2BP2 and may change the role of IGF2BP2 on PCNA_58648_AP. In the low ETV7 expression group, correlation between IGF2BP2 expression and PCNA_58648_AP splicing level was -0.28 , while such correlation became 0.14 when in the high ETV7 expression group. HOXA7, as a modulator of PPARGC1A, enhanced the splicing regulation on PCNA_58648_AP. In the low HOXA7 expression group, correlation between PPAGGC1A expression and PCNA_58648_AP splicing level was 0.23 , while such correlation became 0.51 in the high HOXA7 expression group. In addition, ARNT2 had a similar effect on RBM47, and BHLHE41 had a similar effect on SAMD14 (Figure 5B). Thus, the results showed that differentially expressed TFs changed the role of RBPs on regulating PCNA_58648_AP, and these triplets could be diagnostic and prognostic biomarkers of KIRC. This way of regulation can provide some insights into the dysregulation of splicing results in many diseases, not only KIRC.

A

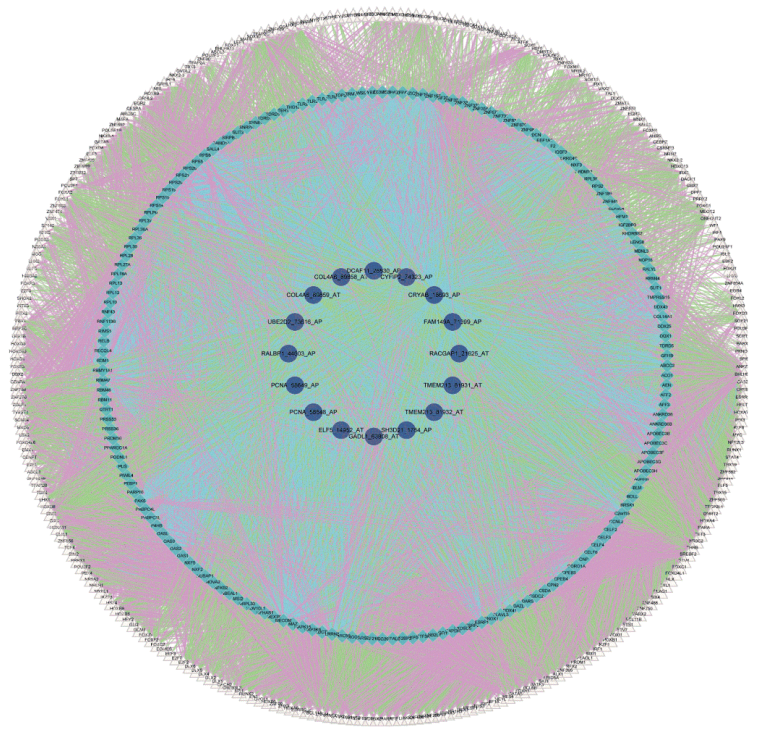

B

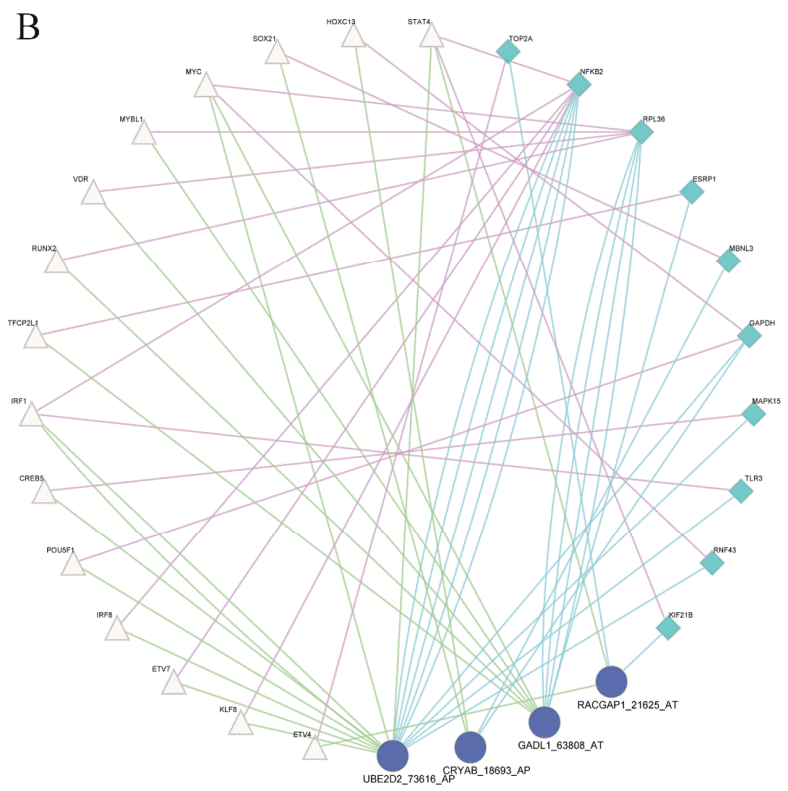

Figure 3. Construction of protein-protein interaction network of triplets. (A) Protein-protein interaction network of 6519 triplets. Pink triangles represent TFs, green rhombuses represent RBPs, purple circles represent ASEs. Purple lines represent relationships between TF and RBP genes, green lines represent relationships between TF genes and ASEs, and blue lines represent the relationships between RBP genes and ASEs. (B) Triplet network involved in known PPI network in STRING. 

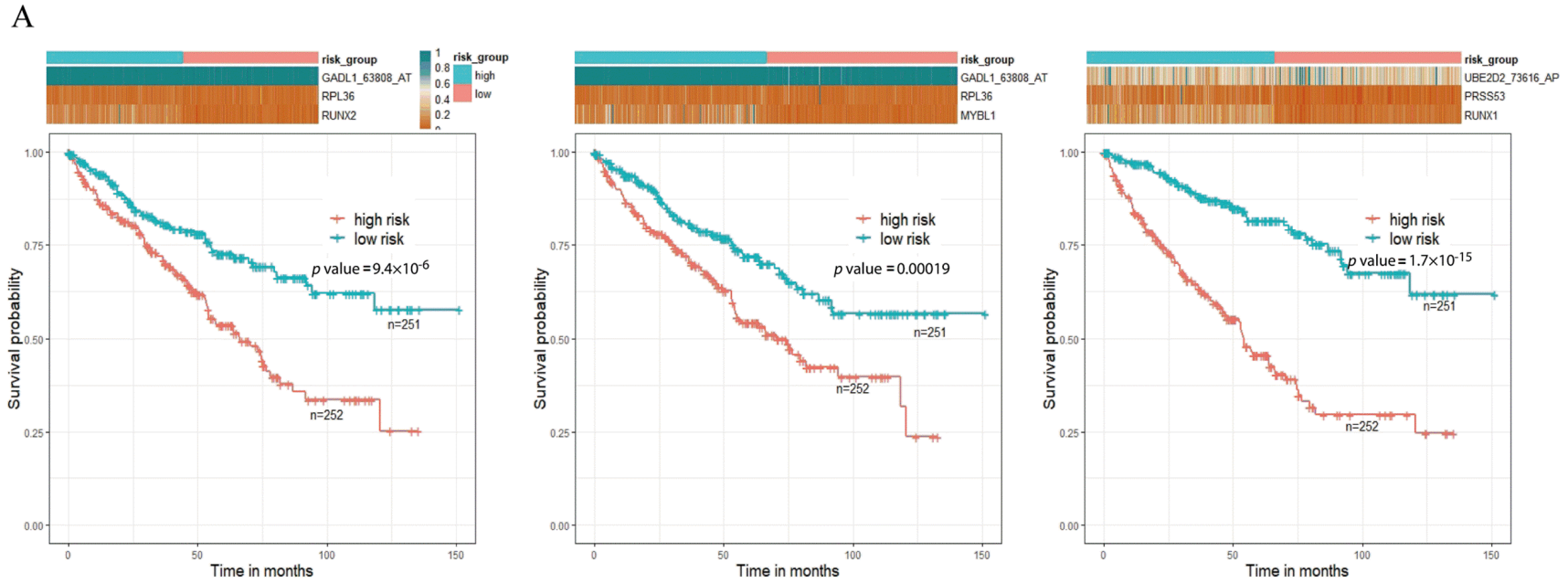

B

C

D
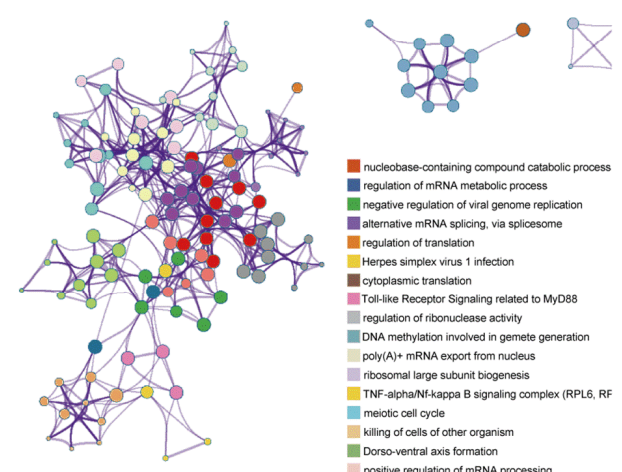

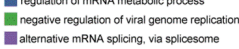

- regulation of translation

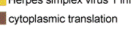

Toll-like Receptor Signaing related to MyDB8

Fillegulation of ribonudease activity

-

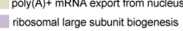

TNF-alphantikappa B signaling complex RRPL6.RF

II meitic cell cyde

-illiling of cells of other organis

positive regulation of mRNA processing

histone H3-k9 metrylation
-
translationa elongation

8
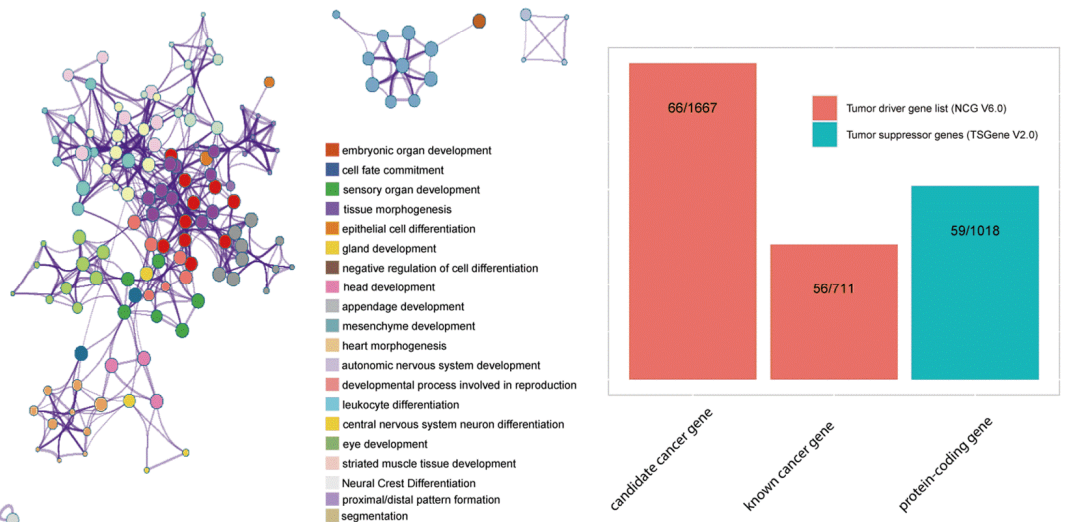

8

Figure 4. Functional analysis of TF-RBP-AS triplets. (A) Survival analysis of three representative triplets. Red line represents the high-risk subgroup, and green line represents the low-risk subgroup. (B) Enrichment analysis of TF genes involved in 6519 triplets. (C) Enrichment analysis of RBP genes involved in 6519 triplets. (D) Cancer-relevant modulators identified according to the Network of Cancer Genes (NCG) and Tumor Suppressor Gene (TSGene) databases (* using Metascape for gene-enrichment analysis). 
A

e.g.
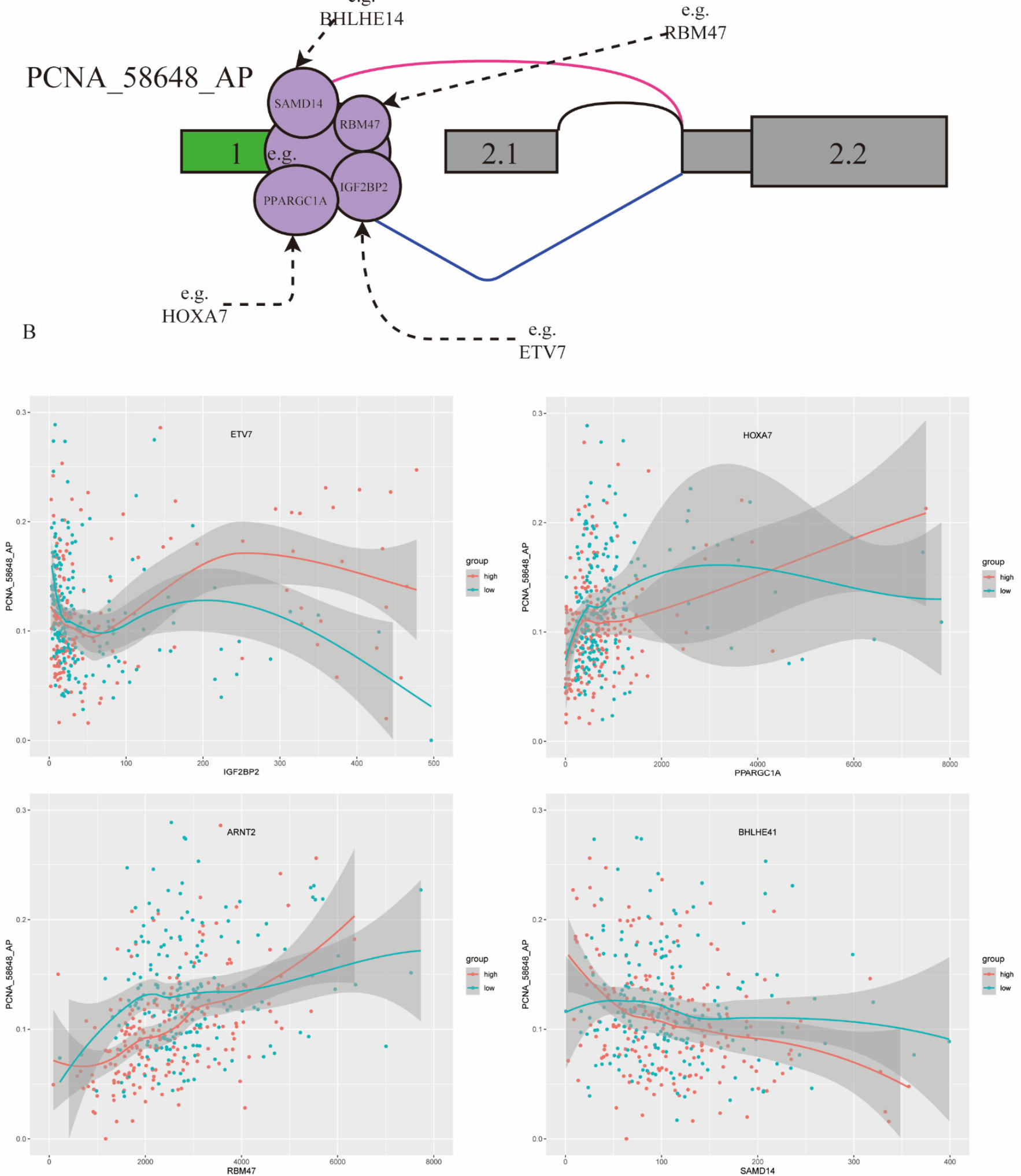

Figure 5. PCNA_58648_AP influenced by TFs and RBPs involved in triplets in KIRC. (A) Examples of some TFs and RBPs involved in triplets. (B) Four triplets influencing splicing of PCNA_58648_AP: four TFs (ETV7, HOXA7, ARNT2, BHLHE41) and four RBPs (IGF2BP2, PPARGC1A, RBM47, SAMD14). Red, samples in high TF expression group (top 40\%); green, samples in low TF expression group (bottom 40\%). X axis is the expression level of RBP, and Y axis is the PSI value of PCNA_58648_AP. 


\section{Discussion}

In this study, we proposed to use a linear mixed-effects model to identify TF-RBP-AS triplets with which the expression level of TFs was associated in changing the targets AS outcomes of RBPs in KIRC. A computational method was previously developed to identify modulators whose expression levels could affect the relationship between the RBPs and its target alternative splicing outcomes, and this only focused on target splicing outcomes of QKI that can be influenced by the expression level of modulators [15]. In addition, several computational methods were developed for identification modulators whose expression levels could affect the regulation activity of TFs toward its target genes [29,30], and these studies reported that the expression level of modulators can affect the transcriptional activities of TFs. The unique ability of our method is that it can identify triplets by considering the influence of some objective factors and discovering how the expression level of TFs is associated with changing the target AS outcomes of RBPs. Our method aimed to discover the impact of TFs on AS after acting on RBPs and provides a new perspective for studying the network of alternative splicing mechanisms in cancers.

Not all ASEs are necessarily related to cancer, so it is an effective way to find a subset of cancer-specific ASEs by comparing the PSI values of the ASE between normal and tumor samples. Then, 33 ASEs corresponding to 23 genes were found by using the method of iterative MI-SIS. A literature review found 10 (TMEM213, ELF5, PCNA, RALBP1, WNK1, SLC17A3, APOC1, SCARB1, DCAF11, CRYAB) out of 23 genes to be related to kidney cancer [28,31-39]. In particular, ELF5, SLC17A3, RALBP1, WNK1, APOC1, and CRYAB were experimentally verified to play an important role in the occurrence and development of kidney cancer. ELF5 is a tumor-suppressor gene for RCC; SLC17A3 is related to the origin of RCC; RALBP1 plays an oncogenic role in RCC; WNK1 promotes renal tumor progression by activating TRPC6-NFAT pathway; APOC1 is significantly correlated with RCC tumor size and histological grade, and CRYAB promotes RCC tumor angiogenesis by increasing vascular survival during tube morphogenesis. On the basis of the role of these genes in kidney cancer, the influence of gene expression on kidney cancer is important. Some of the remaining 13 genes also play important roles in kidney cancer. For example, COL4A6 is related to hereditary nephropathy, and RACGAP1 can promote the proliferation and suppress apoptosis of renal tubular cells $[40,41]$. This evidence indicated that these ASEs are closely related to RCC.

In our study, 302 differentially expressed TFs, 177 differentially expressed RBPs, and 33 cancer-specific ASEs were formed to the combinations of TF-RBP-AS; all of them were screened by linear mixed-effects models to obtain significant TF-RBP-AS triplets for further study. Lastly, 6519 TF-RBP-AS triplets were identified as significant TF-RBP-AS triplets. These triplets included 290 TFs, 175 RBPs, and 16 ASEs corresponding to 13 genes. The results showed that the functions of TFs are very complicated, and even the same TF may play different or opposite roles on the same RBP targets. For example, LYL1 plays an enhanced role on the C2orf15-TMEM213_81931_AT pair, but LYL1 inverts the splicing activity of C2orf15 on RACGAP1_21625_AT. Detailed information can be found in Supplementary Table S2. Results demonstrated the complexity of the alternative splicing regulation mechanism. Among the 16 ASEs involved in 6519 triplets, PCNA_58648_AP received more attention because previous studies showed that PCNA has significant association with RCC. Eighty TF-RBP-PCNA_58648_AP triplets were identified, including 28 TFs and 58 RBPs, and LYL1-RELB-PCNA_58648_AP and HOXA7- PPARGC1A-PCNA_58648_AP were two of them. LYL1 gene amplification was associated with the upregulation of cancer-related pathways in uterine corpus endometrial cancer, and RELB gene plays an oncogenic role in colorectal cancer $[42,43]$. Our results showed that the high expression of LYL1 tended to attenuate RELB regulation role on PCNA_58648_AP. Correlation between RELB expression and PCNA_58648_AP PSI was -0.18 in the low LYL1 expression group, and such correlation changed into -0.06 in the high LYL1 expression group. HOXA7-PPARGC1APCNA_58648_AP was another inferred triplet. HOXA7 is associated with the metastasis of liver cancer [44]. PPARGC1A overexpression promotes lung-cancer metastasis [45]. 
When HOXA7 expression was low, correlation between PPARGC1A expression and the PSI of PCNA_58648_AP was 0.51, while such correlation turned into 0.23 when HOXA7 expression became high.

Two ASEs (i.e., COL4A6_89859_AT and GADL1_63808_AT) in the triplets were the fragments of the targeted RNA of NMD, which is a mechanism to degrade mRNA transcripts containing PTC [19]. COL4A6 and GADL1 are related to cancer. The downregulation of COL4A6 was associated with prostate cancer progression and metastasis, and the overexpression of GADL1 was associated with cancer cell migration and morphology (including cell area, thickness, volume, perimeter length, irregularity, and eccentricity) [46,47]. The expression of COL4A6 and GADL1 may be regulated by alternative splicing-coupled NMD to promote the development of cancer. This evidence indicated that alternative splicing-coupled NMD is related to RCC.

On the basis of the 6519 inferred triplets, we constructed an interaction network using TF-RBP-AS triplets and further refined the network by only including 18 TF-RBPAS triplets that had documented interactions from the STRING database. Some of the interactions had already been reported by other studies. For example, IRF1 could inhibit NFKB2 activity to induce breast cancer cell-specific growth inhibition [48]. MYC can also suppress NFKB2 to accelerate lymphomagenesis, and IRF1 can bind to TLR3 to regulate transcriptional activation in cellular antiviral activities $[49,50]$. Then, the study of the IRF1-NFKB2-UBE2D2 _73616_AP triplets may have discovered their regulatory mechanism in RCC. IRF1 is a tumor-suppressor gene that is associated with RCC [51,52]. NFKB2 is involved in inflammation and immune function and participates in activating the Toll-like receptor 4 (TLR4) signaling pathway [53,54]. The UBE2D2 gene regulates the degradation of misfolded, damaged, or short-lived proteins, which occurs via the ubiquitin (Ub)-proteasome system (UPS), and can inhibit TLR4 signaling [55,56]. The NFKB2 and UBE2D2 genes are related to TLR4. The TLR4 gene plays a fundamental role in pathogen recognition and the activation of innate immunity, is required for IRF1 expression, and the TLR4-IRF1 pathway plays important roles in many diseases [57,58]. UBE2D2 inhibits the expression of IRF1, while NFKB2 promotes the expression of IRF1. When the expression of IRF1 changes from low to high, UBE2D2 and NFKB2 have an enhanced negative correlation trend, which is what we inferred. UBE2D2 and NFKB2 may go through TLR4 to influence IRF1 to destroy the immune system and promote the occurrence and development of cancer. This evidence indicated that interactions in the identified triplets play key roles in the development of RCC and could be drug targets for in RCC.

We performed additional dry lab experiments to prove that the triplets we identified could reveal the mechanism of alternative splicing. We compared interactions in our 6519 identified triplets with protein-protein interactions (PPIs) obtained from the STRING database. The results showed that the 4456 TF-RBP pairs, 1509 TF-ASE pairs, and 1280 RBPASE pairs involved in the triplet had 275 TF-RBP pairs, and 55 TF-ASE pairs and 93 RBPASE pairs overlapped with PPI, respectively, which is shown in Supplementary Figure S2. We also further confirmed interactions in the triplets through the following two aspects. Firstly, we compared the interactions of TF-ASE/RBP in the triplets with known interactions of TF targets. We combined the research results of Zhang [59] and Han [60] on transcription factors and transcription factor target genes and obtained a dataset of 970 transcription factor target genes. This dataset contained 167 transcription factors involved in our triplets. We found that 3815 triplets involved the 167 transcription factors from 6519 triplets and compared them with the 970 transcription factor target genes. The results suggested that RBPs in 163 out of 2593 TF-RBP pairs were confirmed by the 970 transcription factor target genes, and genes corresponding to ASEs in 159 out 861 TF-ASE pairs were confirmed by the 970 transcription factor target genes, which are shown in Supplementary Figure S3. Secondly, in order to verify RBP-ASE pairs, we used the results of Paz's [61] study on the binding site of RBP and RNA. However, only eight RBPs involved in 6519 triplets had information of the binding site from Paz's study, so we only compared 293 triplets involving 64 RBP-ASE pairs with Paz's results. Supplementary Tables S5 and S6 show 
that 24 of 64 RBP-ASE pairs found that RBP had binding sites on ASE. Of the 293 triplets, 145 were in involved in these 24 RBP-ASE pairs, suggesting that the triplets we inferred could reveal the underlying mechanisms of alternative splicing.

In order to investigate whether TF-RBP-AS triplet signatures could act as independent prognostic markers, survival analysis was also performed on each of the triplets on the basis of the results of our constructed prognostic risk score models. Survival analysis results showed that $85.60 \%(5580 / 6519)$ of the triplets were significantly related to the overall survival (OS) of RCC patients. Additionally, although the combination of TF and RBP expression and the PSI value of ASE successfully stratified patients, approximately $83.76 \%$ $(4674 / 5580)$ of the triplets included the component that was not significantly associated with KIRC prognosis. This evidence indicated that these triplets could act as risk predictors of RCC, and network-based biomarkers are expected to be more effective and provide deep insights into the molecular mechanism of RCC progression.

Pathway-enrichment results showed that all TF and RBP genes involved in 6519 triplets were enriched in cancer-development- and progression-related pathways, including herpes simplex virus 1 infection, meiotic cell cycle, and the negative regulation of cell differentiation. Herpes simplex virus 1 (HSV-1) infection is a risk factor in the development of human malignancies which can induce apoptosis in neighboring human cancer cells and can be used as a potential target to improve anticancer activity [62,63]. Meiotic errors of the cell-cycle process are an important characteristic of kidney cancer [64,65], and cell differentiation is involved in the process of many human cancers such as RCC [66,67]. This evidence indicated that these TFs and RBPs are involved in the occurrence and development of RCC and could serve as targets for the treatment of RCC.

Although a model was established in this study, and TFs were integrated into regulatory networks to help improve the understanding of the regulatory network of AS, there are some limitations. Firstly, the roles of triplets acing as a whole need to be further be further confirmed by experiments. Moreover, the function and mechanism of how TF changes RBP regulation on AS need to be further studied by experiments. Lastly, our study can provide a perspective for understanding the regulatory mechanism of alternative splicing in cancer, and the corresponding results are helpful for the treatment for RCC.

\section{Materials and Methods}

\subsection{Data Acquisition and Processing}

Data on mRNA splicing patterns of KIRC were downloaded from the TCGA SpliceSeq portal (https:/ / bioinformatics.mdanderson.org/TCGASpliceSeq) (accessed on 9 February 2020). TCGA SpliceSeq is a web-based bioinformatics resource with information of mRNA splicing patterns of 33 different tumors, which provide the PSI value for each splicing event in each cancer sample [68]. The PSI value uses a ratio to quantify the expression of splicing events [69]. The name of each ASE is composed of the gene symbol, ID number, and splicing type, for example, RACGAP1_21625_AT. In total, 72 normal samples and 533 KIRC samples were enrolled in the analysis of ASEs, and the mean value of each AS in the normal and KIRC samples was used to replace the missing values of each ASE. We also downloaded full clinical follow-up information data of 537 patients through TCGA Data Commons (https:/ / gdc.cancer.gov/) (accessed on 8 October 2020) for the KIRC cohort.

The expression datasets, including 20,501 genes for 72 normal samples and 529 KIRC samples, were downloaded from The Cancer Genome Atlas (TCGA, http:/ / cancergenome. nih.gov/) (accessed on 24 July 2020). After removing genes that did not express in more than $50 \%$ samples, 18,103 genes were left for further study. The list of 1826 RBPs was obtained from hRBPome (http:/ / caps.ncbs.res.in/hrbpome/) (accessed on 28 June 2020), and each RBP had to be reported by at least two studies. The list of 1635 TFs came from a review of human transcription factors [70].

We downloaded protein-protein interaction (PPI) datasets from the STRING database version 10.5 (http:/ / string-db.org) (accessed on 23 November 2020), which included direct (physical) and indirect (functional) interactions. 


\subsection{Identify Cancer-Specific Alternative Splicing Events}

The mutual information-sure independence screening (MI-SIS) method [71] was used to identify cancer-specific ASEs in our curated datasets. The mutual information value between ASE and the classification of samples (i.e., tumor or normal samples) was first computed for each ASE. Then, 50 ASEs with the highest mutual information value were identified. On the basis of the top 50 ASEs, an event set composed of these 50 events was processed to the next screening process by using the iterative sure independence screening (ISIS) with tenfold cross-validation (CV) method, and LASSO as the penalty function for intermediate penalized likelihood estimation to form the final set of those highly related to cancer ASEs. Details of the MI-SIS method can be found in Supplementary Materials.

\subsection{Identification of Differentially Expressed RBPs and TFs}

The expression data of $1826 \mathrm{RBP}$ genes and $1635 \mathrm{TF}$ genes were screened from the mRNA expression profiles. Then, $\log 2 \mathrm{FC}$ and Wilcoxon test were used to select differentially expressed RBPs and TF genes between tumor and normal samples. Only genes with an absolute value of $\log 2 \mathrm{FC}$ larger than $1(\mid \log 2 \mathrm{FCl}>1)$ and adjusted $p$ value $<0.05$ (Wilcoxon test) were selected as differentially expressed RBPs and TFs.

\subsection{Construction of TF-RBP-AS Triplets in KIRC}

In this study, a linear mixed-effects model as shown in (1) was used to build TF-RBPAS triplets. The model was as follows:

$$
\left\{\begin{array}{c}
Y_{A S}=\beta_{0}+\beta_{1} X_{R B P}+\beta_{2} X_{T F}+\beta_{3} X_{R B P} * X_{T F}+\beta_{4} X_{\text {gender }}+\beta_{5} X_{\text {stage }}+b_{1} Z_{\text {age }}+b_{2} Z_{\text {race }}+b_{3} Z_{\text {year }}+\varepsilon \\
b_{i} \sim N(0, D), i=1,2,3, \varepsilon \sim N(0, \Sigma)
\end{array}\right.
$$

where $Y_{A S}$ is the PSI value of an ASE, which is the dependent variable. $X_{R B P}$ and $X_{T F}$ are the expressions of an RBP and a TF, respectively, and $X_{\text {gender }}$ and $X_{\text {stage }}$ are the age at initial pathologic diagnosis and the year of initial pathologic diagnosis, respectively. $X_{R B P}$, $X_{T F}, X_{\text {gender }}$ and $X_{\text {stage }}$ are the fixed effects. $Z_{\text {age }}, Z_{\text {race }}$, and $Z_{\text {year }}$ are age at diagnosis, race, and year of diagnosis, respectively, which are random effects. $\beta_{3}$ represents the interactive effect of RBP and TF. If the interaction of RBP and TF affects AS, then $\beta_{3}$ is expected to be nonzero.

Only when the $p$ values of RBP, TF, and the interaction term of RBP and TF were less than 0.05 , and the coefficient of the interaction term of RBP and TF was nonzero, were such TF-RBP-AS triplets considered to be statistically significant. Details of this study design are illustrated in Figure 6.

\subsection{Protein-Protein Interaction Network Analysis}

The protein-protein interaction network in which all TF-RBP-AS triplets were involved was constructed and plotted by Cytoscape (https:/ / cytoscape.org/) (accessed on 20 November 2020). In addition, the triplet network that was involved in the known PPI network in STRING was plotted by Cytoscape.

\subsection{GO Functional and KEGG Pathway Enrichment Analyses of Genes}

TF and RBP genes contained in TF-RBP-AS triplets were selected to perform gene ontology (GO) and Kyoto Encyclopedia of Genes and Genomes (KEGG) pathway-enrichment analysis using Metascape (https:/ / metascape.org/gp/index.html\#/main/step) (accessed on 4 January 2021). A $p$ value less than 0.05 was statistically significant. 
A Identify key ASEs and differentially expressed TFs and RBPs

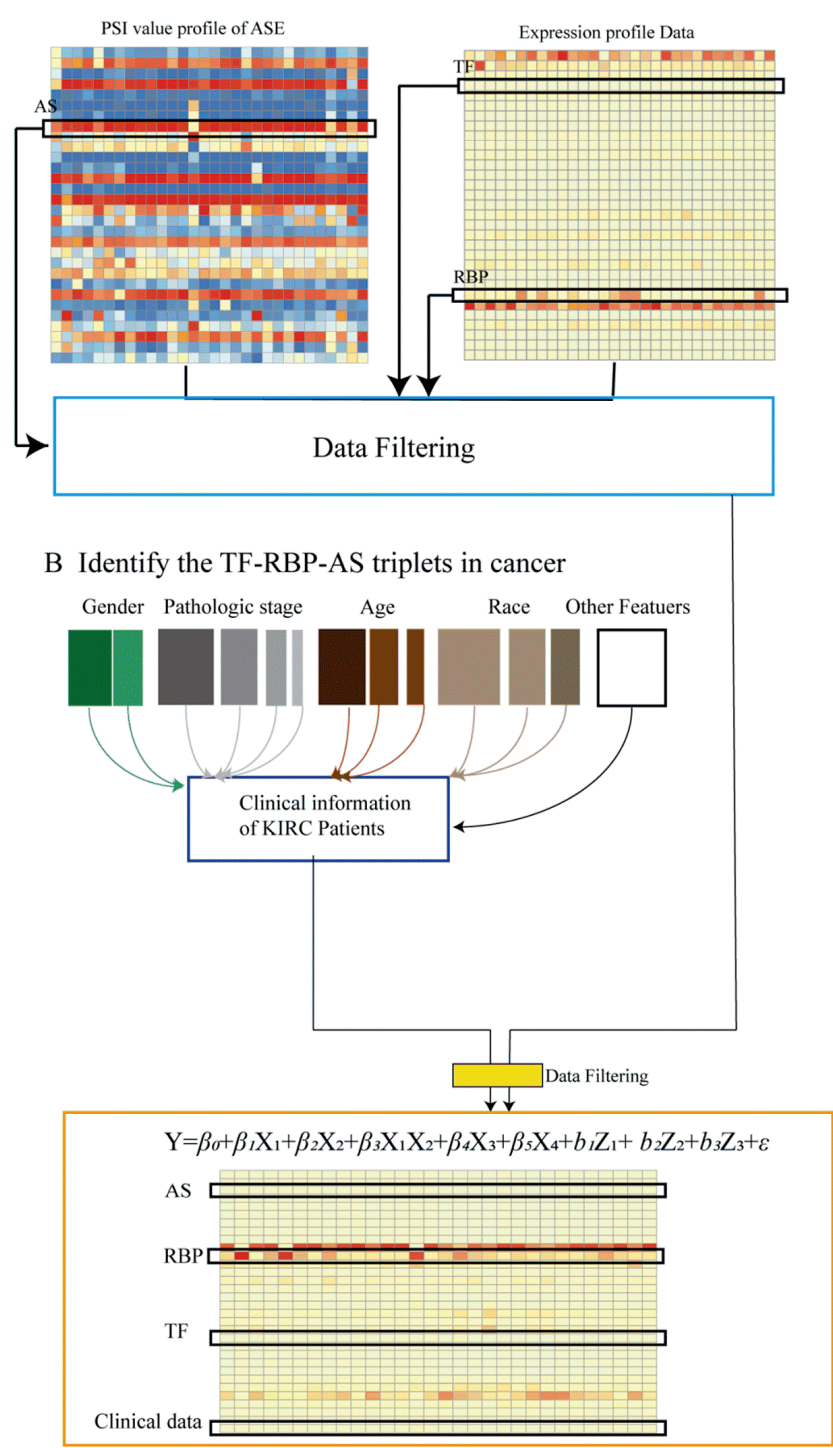

C Permutating the TF expression profiles and calculating the significance of each TF-RBP-AS triplets

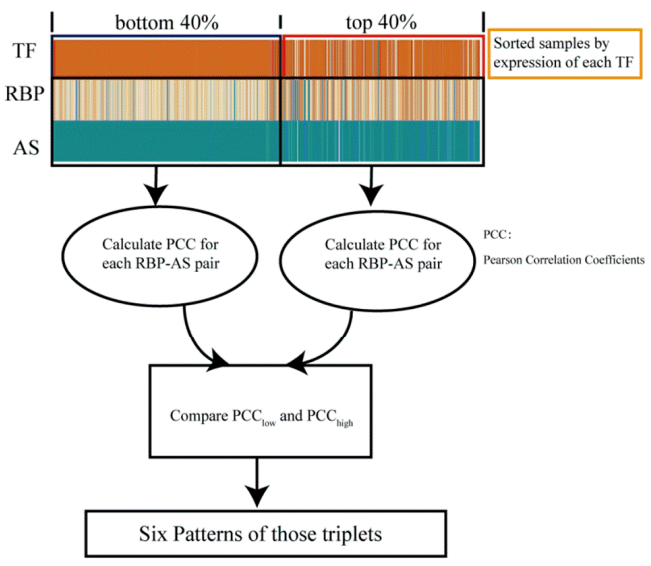

D Profiles of overall triplets in KIRC

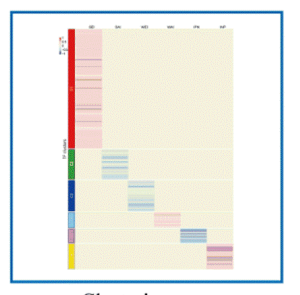

Clustering

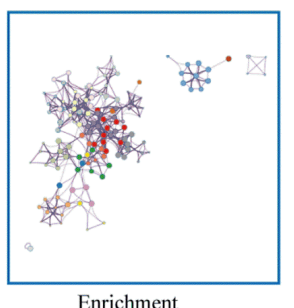

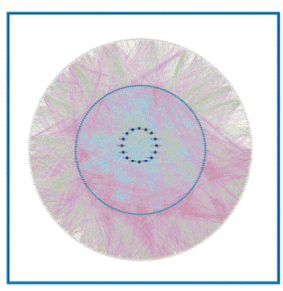

PPI Network

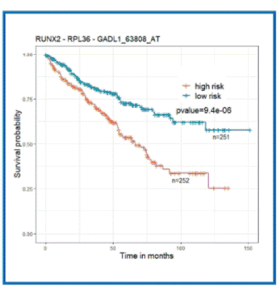

Survival analysis

Figure 6. Research methodology. (A) Key ASEs and differentially expressed RBPs and TFs were identified, expression data of RBP and TF and PSI value of ASE were integrated, relevant clinical information of the patient from the data of TCGA-KIRC was extracted, and all data were integrated. (B) The linear mixed-effects model was used to predict triplets. Only triplets with significant $\beta_{1}, \beta_{2}$, and $\beta_{3} p$ values were considered and selected for the following analysis. Each triplet contains three objects: the expressions of TF and RBP and the PSI value of ASE. (C) For each triplet, we grouped samples into "low" and "high" groups on the basis of the expression level of TF (bottom/top 40\% samples) in the specific triplet, and we compared Pearson's correlation coefficient values of RBP expression and PSI value of ASE in two groups, identifying TF function categories. (D) In order to explore the prognostic value of these triplets, clustering analysis was performed, and a PPI network was constructed; enrichment and survival analyses were carried out.

\subsection{Establishment of Triplet Signature for KIRC Prognosis}

We used the R survival package and coxph function for survival analysis. RBPs, TFs, and ASEs in TF-RBP-AS triplets were integrated into triplet signatures through calculating risk scores with the following formula:

$R S=$ Coefficient of ASE $*$ PSI value of ASE $+\sum_{i=1}^{2}$ Coefficient of Gene $(i) *$ Expression of Gene $(i)$.

where RS is a triplet signature risk score, short for "risk score"; Coefficient of ASE is the regression coefficient of ASE Gene in the model of univariate Cox regression; PSI value of 
ASE is the PSI value of ASE for the sample. Coefficient of Gene is the regression coefficient of the TF or RBP gene in the model of univariate Cox regression; Expression of Gene is the expression value of the TF or RBP gene. On the basis of this formula, each sample could obtain a triplet signature risk score. Then, the median risk score was used as the cutoff to divide patients into high- or low-risk subgroups. A Kaplan-Meier (KM) survival curve was created and the log-rank test was then performed to compare survival between the high- and low-risk groups.

\subsection{Statistical Analysis and Software}

Data were analyzed and visualized using R statistics software version 3.6.1 and ggplot2 package. Correlations were assessed using Pearson's correlation coefficient.

\section{Conclusions}

Our computational method identified TFs whose expression levels could affect the relationship between RBPs and target alternative splicing outcomes. Using this method, we identified 6519 significant TF-RBP-AS triplets, including 290 TFs, 175 RBPs, and 16 ASEs. Regulatory networks constructed for TF-RBP-AS triplets explained the mechanism of the dysregulation of AS by TF-dependent RBP. Pathway enrichment analysis indicated that these triplets were highly correlated with the development of RCC. Therefore, they could be used as therapeutic targets in novel treatment strategies in kidney cancer. Finally, the survival analysis based on risk score models in our study seems to indicate that triplets could also serve in the future as prognostic markers in RCC cases. Future research is needed to confirm this hypothesis and promising data.

Supplementary Materials: The following are available online at https:/ / www.mdpi.com/article/10 .3390/ijms22168789/s1, Figure S1: receiver operating characteristic curve of 33 ASEs; Figure S2: gene pair relationship comparison of PPI and triplets; Figure S3: alignment results of transcription factor target genes; Table S1: details of differentially expressed RBPs in kidney cancer; Table S2: details of differentially expressed TFs in kidney cancer; Table S3: details of 6519 TF-mediated significant triplets; Table S4: detailed information on 5580 triplets with significant survival differences; Table S5: comparison results of eight RBPs with binding sites; Table S6: 145 triplets which RBP has binding sites on ASE. Supplementary Materials: details of iterative MI-SIS method.

Author Contributions: Conceptualization, M.H. and F.H.; methodology, M.H. and F.H.; software, M.H.; validation, M.H. and F.H.; formal analysis, F.H.; investigation, M.H.; resources, F.H.; data curation, M.H. and F.H.; writing-original draft preparation, M.H.; writing-review and editing, F.H.; visualization, M.H.; supervision, F.H.; project administration, F.H.; funding acquisition, F.H. Both authors have read and agreed to the published version of the manuscript.

Funding: This work was supported by grants from the National Natural Science Foundation of China (61903285) and the Fundamental Research Funds for the Central Universities (WUT: 2020IVB031, WUT: 2020IB003, WUT: 2020IA005). Grants-in-Aid supported this study financially only and had no role in the design of the study and collection, analysis, and interpretation of data, or in writing the manuscript.

Institutional Review Board Statement: Not applicable.

Informed Consent Statement: Not applicable.

Data Availability Statement: Not applicable.

Conflicts of Interest: The authors declare no conflict of interest.

\section{References}

1. Ricketts, C.J.; De Cubas, A.A.; Fan, H.; Smith, C.C.; Lang, M.; Reznik, E.; Bowlby, R.; Gibb, E.A.; Akbani, R.; Beroukhim, R.; et al. The Cancer Genome Atlas Comprehensive Molecular Characterization of Renal Cell Carcinoma. Cell Rep. 2018, 23, 313-326.e5. [CrossRef] [PubMed]

2. Yin, L.; Li, W.; Wang, G.; Shi, H.; Wang, K.; Yang, H.; Peng, B. NR1B2 suppress kidney renal clear cell carcinoma (KIRC) progression by regulation of LATS 1/2-YAP signaling. J. Exp. Clin. Cancer Res. 2019, 38, 343. [CrossRef] 
3. Chen, J.; Cao, N.; Li, S.; Wang, Y. Identification of a Risk Stratification Model to Predict Overall Survival and Surgical Benefit in Clear Cell Renal Cell Carcinoma With Distant Metastasis. Front. Oncol. 2021, 11, 630842. [CrossRef]

4. Capitanio, U.; Montorsi, F. Renal cancer. Lancet 2016, 387, 894-906. [CrossRef]

5. Wang, Y.; Chen, Y.; Zhu, B.; Ma, L.; Xing, Q. A Novel Nine Apoptosis-Related Genes Signature Predicting Overall Survival for Kidney Renal Clear Cell Carcinoma and its Associations with Immune Infiltration. Front. Mol. Biosci. 2021, 8, 567730. [CrossRef] [PubMed]

6. Orlandella, R.M.; Turbitt, W.J.; Gibson, J.T.; Boi, S.K.; Li, P.; Smith, D.L., Jr.; Norian, L.A. The Antidiabetic Agent Acarbose Improves Anti-PD-1 and Rapamycin Efficacy in Preclinical Renal Cancer. Cancers 2020, 12, 2872. [CrossRef]

7. Roy, B.; Haupt, L.M.; Griffiths, L.R. Alternative splicing (AS) of genes as an approach for generating protein complexity. Curr. Genom. 2013, 14, 182-194. [CrossRef]

8. Nilsen, T.W.; Graveley, B.R. Expansion of the eukaryotic proteome by alternative splicing. Nature 2010, 463, 457-463. [CrossRef]

9. Le, K.Q.; Prabhakar, B.S.; Hong, W.J.; Li, L.C. Alternative splicing as a biomarker and potential target for drug discovery. Acta Pharmacol. Sin. 2015, 36, 1212-1218. [CrossRef]

10. Wang, Y.; Liu, J.; Huang, B.O.; Xu, Y.M.; Li, J.; Huang, L.F.; Lin, J.; Zhang, J.; Min, Q.H.; Yang, W.M.; et al. Mechanism of alternative splicing and its regulation. Biomed. Rep. 2015, 3, 152-158. [CrossRef] [PubMed]

11. Wang, J.; Wang, C.; Li, L.; Yang, L.; Wang, S.; Ning, X.; Gao, S.; Ren, L.; Chaulagain, A.; Tang, J.; et al. Alternative splicing: An important regulatory mechanism in colorectal carcinoma. Mol. Carcinog. 2021, 60, 279-293. [CrossRef]

12. Zuo, Y.; Zhang, L.; Tang, W.; Tang, W. Identification of prognosis-related alternative splicing events in kidney renal clear cell carcinoma. J. Cell. Mol. Med. 2019, 23, 7762-7772. [CrossRef]

13. Xiao, L.; Zou, G.; Cheng, R.; Wang, P.; Ma, K.; Cao, H.; Zhou, W.; Jin, X.; Xu, Z.; Huang, Y.; et al. Alternative splicing associated with cancer stemness in kidney renal clear cell carcinoma. BMC Cancer 2021, 21, 703. [CrossRef]

14. Gao, L.; He, R.Q.; Huang, Z.G.; Dang, Y.W.; Gu, Y.Y.; Yan, H.B.; Li, S.H.; Chen, G. Genome-wide Analysis of the Alternative Splicing Profiles Revealed Novel Prognostic Index for Kidney Renal Cell Clear Cell Carcinoma. J. Cancer 2020, 11, 1542-1554. [CrossRef]

15. Wang, Y.; Chen, S.X.; Rao, X.; Liu, Y. Modulator-Dependent RBPs Changes Alternative Splicing Outcomes in Kidney Cancer. Front. Genet. 2020, 11, 265. [CrossRef] [PubMed]

16. Ule, J.; Blencowe, B.J. Alternative Splicing Regulatory Networks: Functions, Mechanisms, and Evolution. Mol. Cell 2019, 76, 329-345. [CrossRef] [PubMed]

17. Ray, D.; Kazan, H.; Cook, K.B.; Weirauch, M.T.; Najafabadi, H.S.; Li, X.; Gueroussov, S.; Albu, M.; Zheng, H.; Yang, A.; et al. A compendium of RNA-binding motifs for decoding gene regulation. Nature 2013, 499, 172-177. [CrossRef] [PubMed]

18. Kang, D.; Lee, Y.; Lee, J.S. RNA-Binding Proteins in Cancer: Functional and Therapeutic Perspectives. Cancers 2020, 12, 2699. [CrossRef]

19. Baker, K.E.; Parker, R. Nonsense-mediated mRNA decay: Terminating erroneous gene expression. Curr. Opin. Cell Biol. 2004, 16, 293-299. [CrossRef]

20. Micale, L.; Muscarella, L.A.; Marzulli, M.; Augello, B.; Tritto, P.; D’Agruma, L.; Zelante, L.; Palumbo, G.; Merla, G. VHL frameshift mutation as target of nonsense-mediated mRNA decay in Drosophila melanogaster and human HEK293 cell line. J. Biomed. Biotechnol. 2009, 2009, 860761. [CrossRef]

21. Zheng, S. Alternative splicing and nonsense-mediated mRNA decay enforce neural specific gene expression. Int. J. Dev. Neurosci. 2016, 55, 102-108. [CrossRef]

22. García-Moreno, J.F.; Romão, L. Perspective in Alternative Splicing Coupled to Nonsense-Mediated mRNA Decay. Int. J. Mol. Sci. 2020, 21, 9424. [CrossRef]

23. Hong, M.; Zhang, Z.; Chen, Q.; Lu, Y.; Zhang, J.; Lin, C.; Zhang, F.; Zhang, W.; Li, X.; Zhang, W.; et al. IRF1 inhibits the proliferation and metastasis of colorectal cancer by suppressing the RAS-RAC1 pathway. Cancer Manag. Res. 2019, 11, 369-378. [CrossRef] [PubMed]

24. Morais, C.; Gobe, G.; Johnson, D.W.; Healy, H. The emerging role of nuclear factor kappa B in renal cell carcinoma. Int. J. Biochem. Cell Biol. 2011, 43, 1537-1549. [CrossRef] [PubMed]

25. Voutsadakis, I.A. Ubiquitin- and ubiquitin-like proteins-conjugating enzymes (E2s) in breast cancer. Mol. Biol. Rep. 2013, 40, 2019-2034. [CrossRef]

26. Repana, D.; Nulsen, J.; Dressler, L.; Bortolomeazzi, M.; Venkata, S.K.; Tourna, A.; Yakovleva, A.; Palmieri, T.; Ciccarelli, F.D. The Network of Cancer Genes (NCG): A comprehensive catalogue of known and candidate cancer genes from cancer sequencing screens. Genome Biol. 2019, 20, 1. [CrossRef] [PubMed]

27. Zhao, M.; Kim, P.; Mitra, R.; Zhao, J.; Zhao, Z. TSGene 2.0: An updated literature-based knowledgebase for tumor suppressor genes. Nucleic Acids Res. 2016, 44, D1023-D1031. [CrossRef]

28. Pramanik, S.; Sur, S.; Bankura, B.; Panda, C.K.; Pal, D.K. Expression of proliferating cell nuclear antigen and Ki-67 in renal cell carcinoma in eastern Indian patients. Int. Surg. J. 2019, 6. [CrossRef]

29. Li, J.; Wang, Y.; Rao, X.; Wang, Y.; Feng, W.; Liang, H.; Liu, Y. Roles of alternative splicing in modulating transcriptional regulation. BMC Syst. Biol. 2017, 11, 89. [CrossRef] [PubMed]

30. Li, Y.; Wang, Z.; Wang, Y.; Zhao, Z.; Zhang, J.; Lu, J.; Xu, J.; Li, X. Identification and characterization of lncRNA mediated transcriptional dysregulation dictates lncRNA roles in glioblastoma. Oncotarget 2016, 7, 45027-45041. [CrossRef] 
31. Wrzesinski, T.; Szelag, M.; Cieslikowski, W.A.; Ida, A.; Giles, R.; Zodro, E.; Szumska, J.; Pozniak, J.; Kwias, Z.; Bluyssen, H.A.; et al. Expression of pre-selected TMEMs with predicted ER localization as potential classifiers of ccRCC tumors. BMC Cancer 2015, 15, 518. [CrossRef] [PubMed]

32. Lapinskas, E.J.; Svobodova, S.; Davis, I.D.; Cebon, J.; Hertzog, P.J.; Pritchard, M.A. The Ets transcription factor ELF5 functions as a tumor suppressor in the kidney. Twin Res. Hum. Genet. 2011, 14, 316-322. [CrossRef] [PubMed]

33. Jinesh, G.G.; Kamat, A.M. RalBP1 and p19-VHL play an oncogenic role, and p30-VHL plays a tumor suppressor role during the blebbishield emergency program. Cell Death Discov. 2017, 3, 17023. [CrossRef]

34. Kim, J.H.; Hwang, K.H.; Eom, M.; Kim, M.; Park, E.Y.; Jeong, Y.; Park, K.S.; Cha, S.K. WNK1 promotes renal tumor progression by activating TRPC6-NFAT pathway. FASEB J. 2019, 33, 8588-8599. [CrossRef] [PubMed]

35. Young, M.D.; Mitchell, T.J.; Braga, F.A.V.; Tran, M.G.; Stewart, B.J.; Ferdinand, J.R.; Collord, G.; Botting, R.A.; Popescu, D.M.; Loudon, K.W.; et al. Single- cell transcriptomes from human kidneys reveal the cellular identity of renal tumors. Science 2018, 599, 594-599. [CrossRef] [PubMed]

36. Cui, Y.; Miao, C.; Hou, C.; Wang, Z.; Liu, B. Apolipoprotein C1 (APOC1): A Novel Diagnostic and Prognostic Biomarker for Clear Cell Renal Cell Carcinoma. Front. Oncol. 2020, 10, 1436. [CrossRef]

37. Pospiech, E.; Ligeza, J.; Wilk, W.; Golas, A.; Jaszczynski, J.; Stelmach, A.; Rys, J.; Blecharczyk, A.; Wojas-Pelc, A.; Jura, J.; et al. Variants of SCARB1 and VDR Involved in Complex Genetic Interactions May Be Implicated in the Genetic Susceptibility to Clear Cell Renal Cell Carcinoma. Biomed. Res. Int. 2015, 2015, 860405. [CrossRef] [PubMed]

38. Chen, Y.; He, J.; Su, C.; Wang, H.; Chen, Y.; Guo, W.; Li, Y.; Ding, G. LINC00461 affects the survival of patients with renal cell carcinoma by acting as a competing endogenous RNA for microRNA942. Oncol. Rep. 2019, 42, 1924-1934. [CrossRef]

39. Dimberg, A.; Rylova, S.; Dieterich, L.C.; Olsson, A.K.; Schiller, P.; Wikner, C.; Bohman, S.; Botling, J.; Lukinius, A.; Wawrousek, E.F.; et al. alphaB-crystallin promotes tumor angiogenesis by increasing vascular survival during tube morphogenesis. Blood 2008, 111, 2015-2023. [CrossRef]

40. Murata, T.; Katayama, K.; Oohashi, T.; Jahnukainen, T.; Yonezawa, T.; Sado, Y.; Ishikawa, E.; Nomura, S.; Tryggvason, K.; Ito, M. COL4A6 is dispensable for autosomal recessive Alport syndrome. Sci. Rep. 2016, 6, 29450. [CrossRef]

41. Zhou, W.; Zhao, S.; Xu, S.; Sun, Z.; Liang, Y.; Ding, X. RacGAP1 ameliorates acute kidney injury by promoting proliferation and suppressing apoptosis of renal tubular cells. Biochem. Biophys. Res. Commun. 2020, 527, 624-630. [CrossRef] [PubMed]

42. Kim, S.I.; Lee, J.W.; Lee, N.; Lee, M.; Kim, H.S.; Chung, H.H.; Kim, J.W.; Park, N.H.; Song, Y.S.; Seo, J.S. LYL1 gene amplification predicts poor survival of patients with uterine corpus endometrial carcinoma: Analysis of the Cancer genome atlas data. BMC Cancer 2018, 18, 494. [CrossRef] [PubMed]

43. Zhou, X.; Shan, Z.; Yang, H.; Xu, J.; Li, W.; Guo, F. RelB plays an oncogenic role and conveys chemo-resistance to DLD-1 colon cancer cells. Cancer Cell Int. 2018, 18, 181. [CrossRef] [PubMed]

44. Tang, B.; Qi, G.; Sun, X.; Tang, F.; Yuan, S.; Wang, Z.; Liang, X.; Li, B.; Yu, S.; Liu, J.; et al. HOXA7 plays a critical role in metastasis of liver cancer associated with activation of Snail. Mol. Cancer 2016, 15, 57. [CrossRef] [PubMed]

45. Li, J.D.; Feng, Q.C.; Qi, Y.; Cui, G.; Zhao, S. PPARGC1A is upregulated and facilitates lung cancer metastasis. Exp. Cell Res. 2017, 359, 356-360. [CrossRef]

46. Ma, J.B.; Bai, J.Y.; Zhang, H.B.; Gu, L.; He, D.; Guo, P. Downregulation of Collagen COL4A6 Is Associated with Prostate Cancer Progression and Metastasis. Genet. Test. Mol. Biomarkers 2020, 24, 399-408. [CrossRef]

47. Wu, T.N.; Chen, C.K.; Liu, I.C.; Wu, L.S.; Cheng, A.T. Effects of GADL1 overexpression on cell migration and the associated morphological changes. Sci. Rep. 2019, 9, 5298. [CrossRef] [PubMed]

48. Armstrong, M.J.; Stang, M.T.; Liu, Y.; Yan, J.; Pizzoferrato, E.; Yim, J.H. IRF-1 inhibits NF-kappaB activity, suppresses TRAF2 and cIAP1 and induces breast cancer cell specific growth inhibition. Cancer Biol. Ther. 2015, 16, 1029-1041. [CrossRef]

49. Keller, U.; Huber, J.; Nilsson, J.A.; Fallahi, M.; Hall, M.A.; Peschel, C.; Cleveland, J.L. Myc suppression of Nfkb2 accelerates lymphomagenesis. BMC Cancer 2010, 10, 348. [CrossRef] [PubMed]

50. Ren, G.; Cui, K.; Zhang, Z.; Zhao, K. Division of labor between IRF1 and IRF2 in regulating different stages of transcriptional activation in cellular antiviral activities. Cell Biosci. 2015, 5, 17. [CrossRef]

51. Brandt, M.; Hellmuth, S.K.; Ziosi, M.; Gokden, A.; Wolman, A.; Lam, N.; Recinos, Y.; Hornung, V.; Schumacher, J.; Lappalainen, T. An autoimmune disease risk variant has a trans master regulatory effect mediated by IRF1 under immune stimulation. bioRxiv 2020. [CrossRef]

52. Chen, F.F.; Jiang, G.; Xu, K.; Zheng, J.N. Function and mechanism by which interferon regulatory factor-1 inhibits oncogenesis. Oncol. Lett. 2013, 5, 417-423. [CrossRef]

53. Hayden, M.S.; Ghosh, S. NF-kappaB in immunobiology. Cell. Res. 2011, 21, 223-244. [CrossRef]

54. Kawai, T.; Akira, S. Signaling to NF-kappaB by Toll-like receptors. Trends Mol. Med. 2007, 13, 460-469. [CrossRef] [PubMed]

55. Polge, C.; Attaix, D.; Taillandier, D. Role of E2-Ub-conjugating enzymes during skeletal muscle atrophy. Front. Physiol. 2015, 6. [CrossRef] [PubMed]

56. Qi, X.; Wang, H.; Xia, L.; Lin, R.; Li, T.; Guan, C.; Liu, T. miR-30b-5p releases HMGB1 via UBE2D2/KAT2B/HMGB1 pathway to promote pro-inflammatory polarization and recruitment of macrophages. Atherosclerosis 2021, 324, 38-45. [CrossRef]

57. Sacramento, L.A.; Benevides, L.; Maruyama, S.R.; Tavares, L.; Fukutani, K.F.; Francozo, M.; Sparwasser, T.; Cunha, F.Q.; Almeida, R.P.; da Silva, J.S.; et al. TLR4 abrogates the Th1 immune response through IRF1 and IFN-beta to prevent immunopathology during L. infantum infection. PLoS Pathog. 2020, 16, e1008435. [CrossRef] [PubMed] 
58. Wu, D.; Pan, P.; Su, X.; Zhang, L.; Qin, Q.; Tan, H.; Huang, L.; Li, Y. Interferon Regulatory Factor-1 Mediates Alveolar Macrophage Pyroptosis During LPS-Induced Acute Lung Injury in Mice. Shock 2016, 46, 329-338. [CrossRef]

59. Zhang, Q.; Liu, W.; Zhang, H.M.; Xie, G.Y.; Miao, Y.R.; Xia, M.; Guo, A.Y. hTFtarget: A Comprehensive Database for Regulations of Human Transcription Factors and Their Targets. Genom. Proteom. Bioinform. 2020, 18, 120-128. [CrossRef]

60. Han, H.; Cho, J.W.; Lee, S.; Yun, A.; Kim, H.; Bae, D.; Yang, S.; Kim, C.Y.; Lee, M.; Kim, E.; et al. TRRUST v2: An expanded reference database of human and mouse transcriptional regulatory interactions. Nucleic Acids Res. 2018, 46, D380-D386. [CrossRef] [PubMed]

61. Paz, I.; Kosti, I.; Ares, M., Jr.; Cline, M.; Mandel-Gutfreund, Y. RBPmap: A web server for mapping binding sites of RNA-binding proteins. Nucleic Acids Res. 2014, 42, W361-W367. [CrossRef]

62. Parker, T.M.; Smith, E.M.; Ritchie, J.M.; Haugen, T.H.; Vonka, V.; Turek, L.P.; Hamsikova, E. Head and neck cancer associated with herpes simplex virus 1 and 2 and other risk factors. Oral Oncol. 2006, 42, 288-296. [CrossRef] [PubMed]

63. Stanziale, S.F.; Petrowsky, H.; Adusumilli, P.S.; Ben Porat, L.; Gonen, M.; Fong, Y. Infection with oncolytic herpes simplex virus-1 induces apoptosis in neighboring human cancer cells: A potential target to increase anticancer activity. Clin. Cancer Res. 2004, 10, 3225-3232. [CrossRef] [PubMed]

64. Thomasova, D.; Anders, H.J. Cell cycle control in the kidney. Nephrol. Dial. Transplant. 2015, 30, 1622-1630. [CrossRef] [PubMed]

65. Hascoet, P.; Chesnel, F.; Le Goff, C.; Le Goff, X.; Arlot-Bonnemains, Y. Unconventional Functions of Mitotic Kinases in Kidney Tumorigenesis. Front. Oncol. 2015, 5, 241. [CrossRef]

66. Tun, H.W.; Marlow, L.A.; von Roemeling, C.A.; Cooper, S.J.; Kreinest, P.; Wu, K.; Luxon, B.A.; Sinha, M.; Anastasiadis, P.Z.; Copland, J.A. Pathway signature and cellular differentiation in clear cell renal cell carcinoma. PLoS ONE 2010, 5, e10696. [CrossRef] [PubMed]

67. Ruijtenberg, S.; van den Heuvel, S. Coordinating cell proliferation and differentiation: Antagonism between cell cycle regulators and cell type-specific gene expression. Cell Cycle 2016, 15, 196-212. [CrossRef]

68. Ryan, M.; Wong, W.C.; Brown, R.; Akbani, R.; Su, X.; Broom, B.; Melott, J.; Weinstein, J. TCGASpliceSeq a compendium of alternative mRNA splicing in cancer. Nucleic Acids Res. 2016, 44, D1018-D1022. [CrossRef]

69. Wang, E.T.; Sandberg, R.; Luo, S.; Khrebtukova, I.; Zhang, L.; Mayr, C.; Kingsmore, S.F.; Schroth, G.P.; Burge, C.B. Alternative isoform regulation in human tissue transcriptomes. Nature 2008, 456, 470-476. [CrossRef]

70. Lambert, S.A.; Jolma, A.; Campitelli, L.F.; Das, P.K.; Yin, Y.; Albu, M.; Chen, X.; Taipale, J.; Hughes, T.R.; Weirauch, M.T. The Human Transcription Factors. Cell 2018, 172, 650-665. [CrossRef] [PubMed]

71. Pan, W.; Wang, X.; Xiao, W.; Zhu, H. A Generic Sure Independence Screening Procedure. J. Am. Stat. Assoc. 2019, 114, 928-937. [CrossRef] [PubMed] 